\title{
Implicaciones socioambientales de las nanotecnologías
}

\section{FRANCISCO JAVIER CABALLERO ANGUIANO*}

\section{Las nanotecnologías representan una modalidad de producción de alcances disruptivos}

por sus implicaciones ambientales y para la salud. La manipulación de moléculas y átomos ha abierto un espacio de amplias dimensiones en la fabricación de productos más duraderos o medicamentos más eficaces, pero con riesgos que se conocen parcialmente. Al respecto, se analizan las consecuencias de estas tecnologías al mismo tiempo que se plantea la necesidad de mayor información y regulaciones, y se hace énfasis en el peligro de privilegiar la mercantilización de la salud.

*Estudiante,

Doctorado en Estudios del Desarrollo, Universidad Autónoma de Zacatecas

\section{Introducción}

A fines de la década de 1950 ya se hablaba de la posibilidad de desarrollar una tecnología a escala atómica o molecular. En 1959 Richard Feymann, premio Nobel de Física, planteó el factible escenario de fabricar productos a partir del reordenamiento de átomos y moléculas. Dictó una conferencia en la que se abordaba la manera en que las computadoras que trabajan con átomos manipulados podrían consumir poca energía y hacerlo a una velocidad notable; mencionó, por ejemplo, que podría escribirse la Enciclopedia Británica en la cabeza de un alfiler. Desde la perspectiva de la física la propuesta era una posibilidad real. Se considera, entonces, a 1959 como el inicio de la nanotecnología y a Feymann como su iniciador. En 1986, Eric Drexler publicó Engines of creation. The coming era of nanotechnology ${ }^{1}$

${ }^{1}$ Eric Drexler, Engines of creation. The coming era of nanotechnology, Anchor Press Doubleday, 1986, en http:// xaonon.dyndns.org/misc/engines_of_creation.pdf en el que se abordan las nanotecnologías ${ }^{2}$ desde la dimensión en que hoy las entendemos, y cuyo proceso de utilización intensiva y extensiva surge al finalizar la década de 1980:

La importancia del descubrimiento de estos tipos de moléculas [carbono 60], abrieron ya en el final de los años 1980, un nuevo campo de posibles aplicaciones en la elaboración de nuevos tipos de polímeros, superconductores, estructuras con metales o con otros átomos atrapados dentro de estos agrupamientos de carbono, así como nuevos

${ }^{2}$ Un nanómetro (nm) comprende una mil millonésima de un metro y la nanoescala va desde $100 \mathrm{~nm}$ hasta la escala de los átomos (aproximadamente $0.2 \mathrm{~nm}$ ). En tanto, la nanociencia es el estudio de fenómenos y la manipulación de materiales a escala atómica, molecular y macromolecular, donde las propiedades difieren significativamente de las de mayor escala. Finalmente, se define a las nanotecnologías como el diseño, la caracterización, la producción y la aplicación de estructuras, dispositivos y sistemas mediante el control de la forma y el tamaño a escala nanométrica. Royal Society, Final Report, Nanoscience and nanotechnologies, 2004, en http://www.nanotec.org.uk/report/Nano\%20 report $\% 202004 \% 20$ fin.pdf 
catalizadores, productos farmacéuticos y otras posibles aplicaciones industriales. ${ }^{3}$

No es extraño entender que fueran los físicos quienes hicieran los primeros señalamientos sobre la posibilidad de manipulación de la materia a escalas muy reducidas, ya que precisamente al amparo de la carrera armamentista se hacían frecuentes descubrimientos tecnológicos. La dinámica que el armamentismo impele al sistema capitalista en cuanto a los ingentes recursos y por la acelerada investigación y desarrollo, continúa siendo el motor económico de Estados Unidos hasta la actualidad; de ahí que sea la razón, entre otras importantes contribuciones tecnológicas, de la revolución de las tecnologías de la información y las comunicaciones.

Las expectativas acerca de las nanotecnologías resultan elevadas debido a que se encuentran en sectores variados: en el farmacéutico se utilizan para combatir al cáncer, o en la industria de alimentos para la conservación en periodos más prolongados. Las aplicaciones son innumerables, sus ventajas son evidentes, pero su potencial en términos de afectación al medio ambiente y a la salud es una preocupación ya documentada:

El International Council on Nanotechnology (ICON), una institución de la Universidad de Rice (Estados Unidos) que investiga sobre riesgos de los nanomateriales, tiene un banco de información al respecto. De 2000 a 2010 este banco de datos registró un aumento sostenido de artículos publicados en revistas científicas arbitradas dedicados a analizar los potenciales riesgos de los nanomateriales en la salud humana y/o en el medio ambiente (...) En 2010, que es el último año completo registrado, los artículos científicos publicados llegaron a 563. ${ }^{4}$

\footnotetext{
${ }^{3}$ Mario Quintili, «Nanociencia y nanotecnología... un mundo pequeño», Centro de Estudios en Diseño y Comunicación, núm. 42, 2012, p. 127.

${ }^{4}$ Guillermo Foladori, Fernando Bejarano y Noelia Invernizzi, «Nanotecnología: gestión y reglamentación de riesgos para la salud y medio ambiente en América Latina y el Caribe»,
}

Es posible que la difusión y utilización de las aplicaciones a escalas reducidas no haya sido tan acelerada y publicitada como lo fue la revolución de las computadoras y las tecnologías de la información, pero uno de los motivos primordiales que explica la escasa difusión se refiere a las implicaciones riesgosas del uso de productos y tecnologías a escalas moleculares, porque se desconoce, por lo menos oficialmente, qué empresas las fabrican, qué productos las incorporan y, lo más importante, qué efectos tienen sobre el medio ambiente y la salud humana.

En efecto, la escasa información expresa que el componente de riesgo es proporcional a sus alcances disruptivos en la dimensión económica. La dinámica de crecimiento de las nanotecnologías y sus productos ha soslayado la necesidad de información relativa a sus potenciales nocivos para quienes las fabrican, las consumen y los ecosistemas en los que se vierten sus desechos: la lógica de la ganancia subsume a la preservación del ambiente y de la vida como imperativo para la reproducción de la especie.

La discusión en torno a estos asuntos se encuentra limitada a ciertos espacios especializados, con un eco reducido en la opinión pública internacional, pese a que se trata de un problema de dimensiones globales que puede tener efectos adversos sobre el medio ambiente. El énfasis del análisis de las nanotecnologías se ha colocado en el mejoramiento de los procesos productivos que atienden necesidades de consumo y de salud y que eventualmente pueden incidir de modo positivo en la eficiencia energética. Se acepta en principio que las nanotecnologías utilizan menores cantidades de materia prima y de energía para incorporarse a otros procesos ya sea como materias primas o bienes intermedios. Asimismo, se reconoce que la intensidad energética para fabricar nanomateriales es elevada. De cada lado de la perspectiva que se adopte se

Trabalho, Educação E Saúde, vol. 11, núm. 1, 2013, pp. 145-167, en https://doi.org/10.1590/S1981-77462013000100009 
cuenta con promotores y detractores, aunque prevalece la falta de información oficial.

\section{Implicaciones ambientales}

Hasta ahora la atención de los problemas ambientales, como el cambio climático y el calentamiento global, se ha limitado a promover la utilización de tecnologías «verdes» cuya finalidad es reducir las emisiones de contaminantes al hacer eficientes los procesos, transformar en «biodegradables» algunos productos y promover el tratamiento de residuos. La dificultad fundamental, que es la imposibilidad de desacoplar el crecimiento económico del empleo intensivo y extensivo de combustibles fósiles, sigue sin ser atendido, ya que hacerlo supone la cancelación del crecimiento económico, corazón del sistema capitalista.

Las nanotecnologías, en tanto se supone que usan una menor cantidad de materia prima en la elaboración de productos más eficientes porque se requieren menos unidades para obtener mejores resultados o extender su vida útil, se han considerado como una posibilidad técnica y tecnológica para sustituir procesos productivos tradicionales por otros que consumen menos energía de fuentes fósiles:

La fabricación molecular auto-contenida permitiría el desarrollo rápido de tecnología que no daña al medioambiente y conseguiríamos energía solar almacenable en la gran mayoría de las casas e industrias reduciendo (...) emisiones de ceniza, hollín, hidrocarbonos, $\mathrm{NOx}, \mathrm{CO}_{2}$ y petróleo. ${ }^{5}$

El principio de las nanotecnologías radica en combinar artificialmente átomos y moléculas (las medidas en dimensiones nano son de $1^{-6}$ a $1^{-8}$ milímetros) que al incorporarse como

${ }^{5}$ Mario Quintili, op. cit., p. 129. materias primas o bienes intermedios en manufacturas de mayor tamaño adquieren propiedades nuevas, distintas a las que tendrían en dimensiones «normales». Además, a partir de cantidades menores de materias primas se pueden desarrollar procesos y productos más eficientes. Por ello es viable suponer que cuentan con el potencial para reducir el consumo de energía de fuentes fósiles y que en alguna medida
El principio de las nanotecnologías radica en combinar artificialmente átomos y moléculas para desarrollar procesos y productos más eficientes. Ello tiene potencial para reducir el consumo de energía de fuentes fósiles, lo que en las circunstancias climáticas actuales comprende un propósito universal. pueden disminuir las emisiones de $\mathrm{CO}_{2}$, lo que en las circunstancias climáticas actuales se ha convertido en un propósito universal.

Desde la perspectiva de un escenario de reducción de emisiones, las nanotecnologías poseen un amplio potencial para la eficiencia energética, la optimización en los procesos productivos y en la creación de opciones de un desarrollo sustentable. La existencia de excedentes de materias primas (por ejemplo el carbono) que se usan en la elaboración de nanotubos de carbón, uno de los materiales más empleados en la fabricación de productos intermedios y de consumo, permite abrigar expectativas acerca de una utilización menos intensiva de recursos naturales, aspecto que también podría contribuir a la sustentabilidad ambiental, lo que no es limitante para que existan implicaciones medioambientales de otro tipo.

Resulta pertinente acotar que las afectaciones de las nanotecnologías al medio ambiente no sólo se limitan a las dimensiones macro, es decir, a las emisiones de gases de efecto invernadero y a las cuestiones de uso intensivo de materias primas, los problemas del manejo de residuos (en tanto componentes principales de la contaminación de aire y agua), sino al medio ambiente de los organismos vivos, desde componentes de los microambientes (como la cadena trófica) o a nivel molecular y atómico dentro del cuerpo humano. La dimensión ambiental que aquí se postula alude a las dimensiones micro y macro; sin embargo, 
el énfasis se otorga a la primera debido a los problemas de información que serán abordados con posterioridad.

A fin de dar una respuesta tentativa a tales cuestiones, es preciso exponer interrogantes adicionales en términos más puntuales: ¿cuál es el consumo de energía en todo el ciclo de vida de las nanomanufacturas y de los nanomateriales? ¿Se puede plantear que existe ahorro energético desde las materias primas hasta la disposición de residuos? Otra cuestión tiene que ver con el impacto en el medio ambiente y en la salud: ya que no se conocen con precisión en documentos oficiales las interacciones de las nanopartículas con otros seres microscópicos y con la propia naturaleza orgánica de los seres vivos, ¿cuál es el potencial tóxico de las nanopartículas y los nanomateriales en los seres humanos, concretamente en los trabajadores directos, en los científicos que las manipulan y en los consumidores, inclusive después de su uso final?

Desde la perspectiva de dichos planteamientos resulta evidente que hay más preguntas que explicaciones en torno a las nanotecnologías y a sus implicaciones para el medio ambiente y la salud. En diversos estudios y por distintos actores se delinean algunas respuestas, cuya orientación se centra en la toxicidad de estas tecnologías y sus productos. Así, y debido a que de manera creciente han aparecido productos que incorporan nanomanufacturas en su composición, el riesgo medioambiental parece ir en ascenso.

De modo paralelo, pero a menor velocidad, se aprecia un esfuerzo internacional para identificar y regular los riesgos. Sin información confiable los peligros se incrementan y las eventuales soluciones se rezagan. No se trata de afirmar que con motivo de la toxicidad estas tecnologías son nocivas en su totalidad, pese a que ha prevalecido una visión cortoplacista fincada en la rentabilidad que, a diferencia de otros procesos, puede generar una catástrofe ambiental de dimensiones incalculables.

\section{Implicaciones sociales}

Un ámbito de dicha problemática es la vertiente socioeconómica, en la que es relevante examinar las implicaciones de los procesos de investigación y desarrollo (I+D) en países que, como México, se encuentran en condiciones restrictivas de financiamiento público y a la par se considera estratégico este sector, en tanto herramienta de potencial de crecimiento para insertar a las empresas en las cadenas globales de valor (CGV) más dinámicas. Si bien existen deterioros ambientales y de salud asociados con las nanotecnologías, no es posible mantenerse al margen de los procesos que podrían sostener una estrategia de crecimiento. Ante la ausencia de un programa de política industrial, tal enfoque permite ubicar a cada empresa y sector en una dimensión más amplia y facilita la elaboración de programas de inversión e I+D, que a su vez deben acompañarse de una orientación específica en áreas educativas:

The GVC [CGV] framework focuses on the sequences of value added within an industry, from conception to production and end use. It examines the job descriptions, technologies, standards, regulations, products, processes, and markets in specific industries and places, thus providing a holistic view of global industries both from the top down and the bottom up. ${ }^{6}$

Para los países en desarrollo tal circunstancia contiene varios problemas. Como sucede con las tecnologías más avanzadas, por lo general se producen en los países industrializados y están resguardadas por sistemas de protección de la propiedad industrial y elevados costos que complican su acceso. Además, se trata de tecnologías que incorporan un componente de conocimiento

\footnotetext{
${ }^{6}$ Gary Gerefy y Karina Fernandez Stark, «Global value chain analisys: a primer», Center on Globalization, Governance \& Competitiveness, Duke University, 2011, p. 2, en https://globalvaluechains.org/publication/global-value -chain-analysis-primer-2nd-edition
} 
superior y una reducida participación de mano de obra; no obstante, aun en el caso de desarrollarse en una economía subdesarrollada, ello no garantiza mejores condiciones de vida para trabajadores y habitantes.

En un contexto de problemas financieros para el fomento de la inversión en I+D, incorporarse a los sectores de punta brinda la oportunidad de profundizar la brecha tecnológica entre ambos grupos de países. Dicha situación no es nueva, pues desde los 1970 el crecimiento económico enfrenta dos problemas: recursos financieros limitados para la inversión en tecnologías e insuficiente infraestructura en I+D que hace poco factible su integración a la cadena de valor del sector, lo que explica la prevalencia de la comercialización antes que la producción de nanomateriales.

Una consideración clave sobre la incorporación de los países en desarrollo a la fabricación de materias primas, bienes intermedios o productos de consumo basados en nanotecnologías se establece en torno a que sólo lo hacen en la modalidad de comercializadores y no de productores. A pesar de lo anterior, incorporarse en algunos de los eslabones de las cadenas globales de valor es importante para no quedar absolutamente marginado de las tecnologías que encabezan el crecimiento económico en el futuro inmediato y que desde esa perspectiva facilitarán la ejecución de los programas de desarrollo económico y combate a los problemas de marginación y pobreza:

For many countries, especially low-income countries, the ability to effectively insert themselves into GVCs is a vital condition for their development. This supposes an ability to access GVCs, to compete successfully and to «capture the gains» in terms of national economic development, capability building and generating more and better jobs to reduce unemployment and poverty. ${ }^{7}$

${ }^{7}$ Idem.
Si en términos generales la incorporación a las cadenas globales de valor abre la posibilidad de generar y ampliar las opciones de crecimiento y desarrollo para los países subdesarrollados, lo cierto es que también despliega otros retos, en especial los relativos al tipo de mano de obra que se requiere. La calificación que supone producir y manipular nanotecnologías y sus materiales exige la creación de centros de educación y laboratorios con esas especialidades, lo que promoverá la fundación de enclaves educativos y tecnológicos o islas del conocimiento. ${ }^{8}$

\section{Implicaciones productivas}

Las nanotecnologías, en su vertiente productiva, cumplen con las dos condiciones de eficiencia en el uso de los recursos sin reducir el volumen del producto final, e inclusive lo incrementan. Asimismo, se han planteado como una alternativa para crear empleos, sin menoscabo de que trabajadores y consumidores corren riesgos con su manipulación y consumo. Las nanotecnologías tienen el potencial de aplicarse a cualquier sector o producto, como materia prima, producto intermedio o para consumo final. El inicio de la cadena productiva parte de la industria química, pero su potencial adquiere amplitud porque su desarrollo se realiza desde materias primas de gran disponibilidad, con todo y que su conversión a la dimensión nano precisa de una compleja tecnología de baja disponibilidad.

Si bien dicha condición es restrictiva para el acceso a esas tecnologías, hay un creciente interés de los gobiernos (en particular en América Latina, donde se considera un sector estratégico), lo que implica que los escasos recursos financieros tendrán como propósito alimentar la inversión y el desarrollo que converja en las

${ }^{8}$ Edgar Záyago Lau y Mark Rushton, «Nanotecnologías para el desarrollo en Latinoamérica», en Guillermo Foladori y Noela Invernizzi (coords.), Las nanotecnologías en América Latina, México, Universidad Autónoma de Zacatecas/Miguel Ángel Porrúa, 2008, p. 18. 
consumen habitualmente. Se estima que en 2006 existían 360 productos nanotecnológicos ${ }^{13}$ para uso final; en 2016 la cantidad se multiplicó por cinco y se calculaban poco más de mil 800 productos, ${ }^{14}$ cifra que parece ir en constante aumento.

Es necesario esclarecer que existen aplicaciones con efectos positivos en la eficiencia energética. Se trata de la fabricación de celdas solares, celdas de combustible y baterías ecológicas en cuya manufactura se utilizan nanomateriales. Un caso ilustrativo de su potencial se encuentra en la tecnología que incrementa en 200 por ciento la producción de biogás con la introducción de nanopartículas de óxido de hierro en el tratamiento de residuos orgánicos. ${ }^{15}$

\section{Implicaciones en la salud}

Puesto que las nanopartículas reaccionan de manera distinta a la forma en la que actúan en tamaños convencionales, existe el riesgo potencial de reacciones desconocidas cuando entran en contacto con sustancias del medio ambiente. Si bien las nanopartículas pueden no ser tóxicas para el uso humano (lo cual no puede descartarse en todas las dimensiones de lo que se considera como nanopartículas), se desconocen los impactos para otras formas de vida (por ejemplo, los microorganismos, en especial aquellos que forman parte de una cadena trófica). Los daños sobre la salud no son producto de especulaciones sino de casos documentados que han ido en ascenso:

La NanoCeo (Nanotechnology Citizen Engagement Organization) elabora un banco de datos que permite clasificar los artículos científicos sobre riesgos

\footnotetext{
${ }^{13}$ En https://nanotecnologia.fundaciontelefonica.com/2006/ 11/24/productos-nanotecnologicos-inventario-a-fecha-de-hoy/ ${ }^{14}$ Guillermo Foladori, Noela Invernizzi y Edgar Záyago Lau (eds.), Investigación y mercado de nanotecnologías en América Latina, México, Universidad Autónoma de Zacatecas/ Miguel Ángel Porrúa, 2016, p. 5.

${ }^{15}$ En https://www.nanomercado.com/triplican-la-produccion -de-biogas-con-nanoparticulas-de-oxido-de-hierro/
}

de los nanomateriales según el tipo de material nanofacturado (...) entre el año 2000 y finales de 2010 se han acumulado 176 artículos sobre riesgos de los nanotubos de carbono, 190 sobre riesgos de la nanoplata y 70 sobre riesgos del dióxido de titanio, entre otros materiales. ${ }^{16}$

Con la finalidad de extender la información de los peligros asociados con las nanopartículas, una modalidad de análisis se encuentra en el enfoque del ciclo de vida, que considera los procesos de investigación, fabricación, almacenamiento, distribución y residuos generados, y enfatiza la información que se debe proporcionar al consumidor:

Un sistema o ciclo de vida del producto puede empezar con la extracción de materias primas y la generación de energía. Así, los materiales y la energía se convierten en procesos de manufactura, transporte y uso del producto (...) y posterior reciclaje, reuso o desecho. Adoptar un enfoque de ciclo de vida significa reconocer la manera en que nuestras elecciones influyen cada etapa del proceso y así sopesar las ventajas y desventajas, contribuyendo a la economía, el medio ambiente y la sociedad. ${ }^{17}$

A diferencia del concepto de cadena de valor, ${ }^{18}$ que se refiere propiamente al proceso de producción y a todos sus componentes, el ciclo de vida comprende desde la gestión de las materias primas en su estado natural y sus impactos en el medio ambiente y el manejo de residuos hasta que el consumidor final ya no utiliza el producto; su propósito es hacer visibles las afectaciones de cada proceso y a los grupos sociales expuestos en los mismos, con lo que se logra el

\footnotetext{
${ }^{16}$ Guillermo Foladori, Fernando Bejarano y Noela Invernizzi, op. cit., p. 152.

${ }^{17}$ Programa de las Naciones Unidas para el Medio Ambiente, ¿Por qué adoptar un enfoque de ciclo de vida?, ONU, 2004, p. 6, en https://sustainabledevelopment.un.org/content/documents /1731Why_take_a_life_cycle_approach_ES.pdf ${ }^{18}$ Gary Gerefy y Karina Fernandez Stark, op. cit.
} 
involucramiento de los potenciales afectados. ${ }^{19}$ Cada fase del ciclo de vida, que actualmente pone énfasis en el uso de energía fósil, puede ampliarse con la intención de evaluar el daño ambiental y de salud en las dimensiones en las que actúan las nanotecnologías y las nanopartículas; cada una contaría con información suficiente para determinar un cierto nivel de toxicidad y aplicar el principio de precaución.

El principio de precaución ${ }^{20}$ ha sido propuesto en reuniones auspiciadas por la Organización Mundial de la Salud (OMS). Su objetivo es establecer medidas preventivas incluso si se carece de la evidencia directa de que una acción o política pueda causar daño; es decir, con la presunción debería ser suficiente para aplicarlo. En ese sentido, la utilidad de los análisis de ciclo de vida resulta fundamental en la descomposición de las fases de la producción y el consumo en las que se incorporan las nanotecnologías, al igual que en la aplicación de un enfoque de esta naturaleza y no sólo del producto como un todo.

Dicho enfoque advierte la importancia asignada a la necesidad de conocer e informar de los efectos potencialmente dañinos en el medio ambiente y los efectos realmente tóxicos en la salud. En cada proceso hay alguna fase tóxica para científicos, trabajadores y consumidores; se parte de la premisa de que se desconocen las consecuencias de las nanotecnologías en el medio ambiente y la salud. No existe algo semejante a prácticas seguras en las modalidades de eliminación de los productos manufacturados y en el manejo y reciclaje de sus residuos.

Las nanopartículas manufacturadas tienen comportamientos moleculares que amplían sus usos, inclusive pueden realizar más funciones que a un nivel mayor. Tal circunstancia los convierte en un arma de dos filos: su potencial de

${ }^{19}$ Guillermo Foladori, Fernando Bejarano y Noela Invernizzi, op. cit., p. 158.

${ }^{20}$ Guillermo Foladori, Anwar Hasmy, Noela Ivernizzi y Edgar Záyago Lau (coords.), Nanotecnologías en América Latina: trabajo y regulación, México, Universidad Autónoma de Zacatecas/Miguel Ángel Porrúa, 2015, p. 7. mejora es casi tan desconocido como su toxicidad para las personas. Los desarrollos de la nanociencia se colocan en el mercado y no se les asigna información suficiente concerniente a sus riesgos; en ocasiones ni siquiera se consigna que se trata de un producto con algún componente nano y menos de sus peligros. Existen grupos que trabajan al amparo de la Organización de las Naciones Unidas (ONU) a fin de promover el Enfoque Estratégico para la Gestión de Productos Químicos a Nivel Internacional (SAICM, por sus siglas en inglés), cuyo primer mandato es «establecer un sistema mundialmente armonizado de clasificación y etiquetado en el área de riesgos para la salud y medio ambiente», ${ }^{21}$ lo que evidencia el problema de información.

Como sucede con las iniciativas de naturaleza similar, su capacidad real para la implementación forzosa de mecanismos regulatorios está acotada, pese a que en la Cumbre Mundial para el Desarrollo Sostenible se reconoció implícitamente su potencial tóxico y se llamó a la comunidad internacional - de la que México forma parte - para que en 2020 los productos químicos sean elaborados y utilizados con criterios que minimicen los impactos negativos:

[Alcanzar el] objetivo acordado por la Cumbre Mundial sobre el Desarrollo Sostenible celebrada en Johannesburgo en 2002 de asegurar que, para el año 2020, los productos químicos sean elaborados y utilizados de manera que se minimicen los impactos negativos significativos sobre el medio ambiente y la salud humana. ${ }^{22}$

${ }^{21}$ Inter-Organization Programme for the Sound Management of Chemicals (IOMC), «Informe sobre las actividades del programa Interinstitucional para la Gestión Racional de los Productos Químicos (IOMC) y sus Organizaciones Participantes y Observadoras, en la Implementación del Enfoque Estratégico para la Gestión de los Productos Químicos a Nivel Internacional (SAICM)», 2009, p. 4, en http://www.who.int/ iomc/saicm/saicm_impl_spanish.pdf

${ }^{22}$ Idem. 


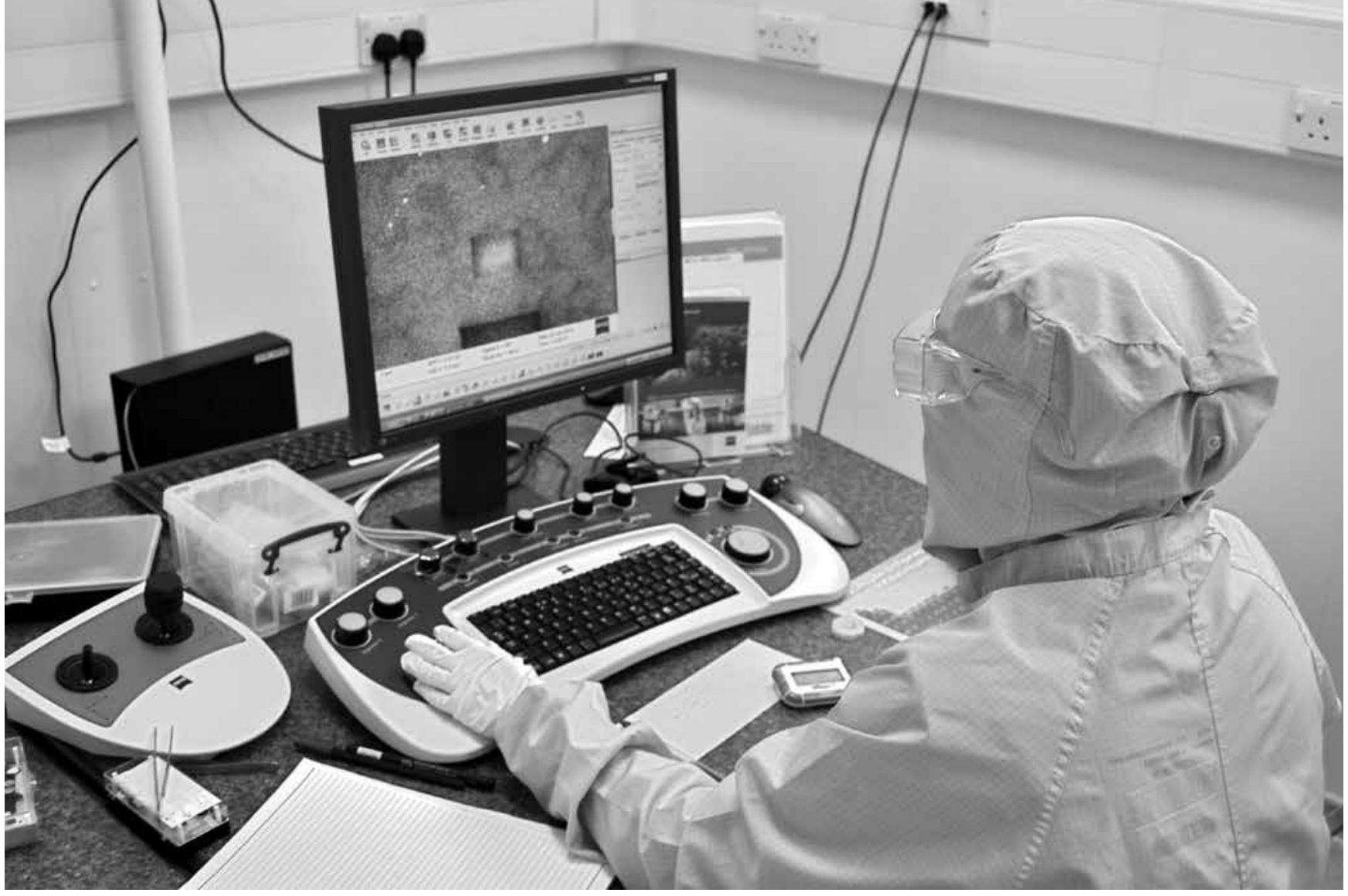

Las nanopartículas manufacturadas

tienen un

comportamiento

molecular que

las convierte en arma de doble filo:

el potencial de

mejora es casi tan desconocido como la toxicidad.
En efecto, en el sector químico y toda su cadena (petroquímica básica y secundaria) se generan las materias primas que después serán componentes de los productos nanomanufacturados. Las implicaciones, en estos términos, se vinculan con la urgencia de proporcionar información clara sobre los peligros del uso de los materiales nano y de la necesidad de regular prácticas asociadas con su producción.

\section{Conclusiones}

Las nanotecnologías están cambiando la configuración productiva y es probable que las modificaciones sean irreversibles, con alcances de naturaleza conflictiva entre las ventajas de más y mejores productos que faciliten la vida de los consumidores que pueden tener acceso a ellos y el potencial negativo sobre el medio ambiente y la salud. Desde esa última dimensión, diversos estudios evidencian la toxicidad y los riesgos relacionados con átomos y moléculas manipulados, situación que se complica ante la falta de información y la ausencia de regulaciones que respeten el principio de precaución. Los actores que producen estas tecnologías tienden a concentrarlas (como ocurre en todos los espacios de las tecnologías más avanzadas), lo que amenaza con excluir a los países subdesarrollados, sin importar que en la perspectiva de las cadenas de valor existe un espacio para su incorporación.

Aunque su condición disruptiva representaba en alguna medida una promesa para un manejo eficiente de recursos, optimización de los residuos y democratización de sus beneficios, lo cierto es que hasta hoy ha prevalecido la lógica de la ganancia. En lo inmediato, parece que la gobernanza global, que ha vislumbrado los riesgos, puede ser el contrapeso para que, a través de la información y las regulaciones, estas tecnologías no se conviertan en un componente que agudice la crisis civilizatoria. 